\title{
WEIGHTED NORM INEQUALITIES FOR THE CONJUGATE FUNCTION ON a-ADIC SOLENOIDS
}

\author{
Nakhlé Asmar and Phyllis Panman
}

\begin{abstract}
In this paper we generalize a theorem of Hunt, Muckenhoupt, and Wheeden on weighted norm inequalities for the conjugate function. Our generalization to the cases of a-adic solenoids is formulated in terms of the ergodic $A_{p}$-condition.
\end{abstract}

\section{Introduction}

We consider an arbitrary noncyclic subgroup of $\mathbb{Q}$ and its compact dual group $\Sigma_{\mathbf{a}}$. There is an explicit construction for $\Sigma_{\mathbf{a}}$ which is called the a-adic solenoid. Since $\widehat{\Sigma_{\mathbf{a}}}$ is simply a subgroup of $\mathbb{Q}, \widehat{\Sigma_{\mathbf{a}}}$ inherits the order from $\mathbb{Q} ;$ that is, if we let $P=\widehat{\Sigma_{\mathbf{a}}} \cap(0, \infty)$ then $P$ defines the order on $\widehat{\Sigma_{\mathbf{a}}}$. For $f \in \mathfrak{L}_{2}\left(\Sigma_{\mathbf{a}}\right)$, we use the Fourier transform of $f$ to define the conjugate function $\tilde{f}$ (with respect to the order $P$ ):

$$
\tilde{f}^{\wedge}(\chi)=-i \operatorname{sgn}_{P}(\chi) \hat{f}(\chi) \quad\left(\chi \in \widehat{\Sigma_{\mathbf{a}}}\right)
$$

where $\operatorname{sgn}_{P}(\chi)=-1,0$, or 1 according to $\chi \in(-P) \backslash\{0\}, \chi=0$, or $\chi \in P \backslash\{0\}$. The operator $f \mapsto \tilde{f}$ is clearly a norm-decreasing multiplier on $\mathfrak{L}_{2}\left(\Sigma_{\mathbf{a}}\right)$. If $1<p<\infty$, the operator $f \mapsto \tilde{f}$ extends from $\mathfrak{L}_{2}\left(\Sigma_{\mathbf{a}}\right) \cap \mathfrak{L}_{p}\left(\Sigma_{\mathbf{a}}\right)$ to a bounded linear operator of $\mathfrak{L}_{p}\left(\Sigma_{\mathbf{a}}\right)$ such that the identity (1.1) holds, and the inequality

$$
\|\tilde{f}\|_{p} \leq M_{p}\|f\|_{p}
$$

holds for all $f \in \mathfrak{L}_{p}\left(\Sigma_{\mathbf{a}}\right)$, where $M_{p}$ is independent of $f$ (see [3], or [1, Theorem 7.2]). We ask for which measures, other than Haar measure, is the operator $f \mapsto \tilde{f}$ a bounded operator. More precisely, if $1<p<\infty$, we seek to characterize those finite nonnegative Borel measures $\nu$ for which the operator $f \mapsto \tilde{f}$ is bounded from $\mathfrak{L}_{p}\left(\Sigma_{\mathbf{a}}, \nu\right) \cap \mathfrak{L}_{1}\left(\Sigma_{\mathbf{a}}\right)$ into $\mathfrak{L}_{p}\left(\Sigma_{\mathbf{a}}, \nu\right)$.

By way of background, we recall that Forelli [7] studied this problem in the case $G=$ $\mathbb{T}$ (henceforth, $\mathbb{T}$ is parameterized by $[-\pi, \pi)$ ). He showed that if the operator $f \mapsto \tilde{f}$ is bounded from $\mathfrak{L}_{p}(\mathbb{T}, \nu) \cap \mathfrak{L}_{1}(\mathbb{T})$ into $\mathfrak{L}_{p}(\mathbb{T}, \nu)$, then $\nu$ must be absolutely continuous with respect to Lebesgue measure $\lambda(\nu \ll \lambda)$, and hence there is a nonnegative function $w$ in $\mathfrak{L}_{1}(\nu)$ where $d \nu=w \frac{d t}{2 \pi}$. This result was later extended by Hunt, et al. [13], who showed that the operator $f \mapsto \tilde{f}$ is bounded from $\mathfrak{L}_{p}(\mathbb{T}, w) \cap \mathfrak{L}_{1}(\mathbb{T})$ into $\mathfrak{L}_{p}(\mathbb{T}, w)$ exactly when $w$ satisfies a property called the $A_{p}$-condition. We state this result in the following definition and theorem:

Definition 1.1. (The $A_{p}$-condition on $\mathbb{T}$ ) Let $1 \leq p<\infty$. Let $w$ be a nonnegative $2 \pi$-periodic measurable function. The function $w$ satisfies the $A_{p}$-condition on $\mathbb{T}$ if

Received February 13, 1995, revised August 14, 1996.

1991 Mathematics Subject Classification: Primary 43A15; Secondary 42A50. 
there is a constant $A_{p}$ independent of all intervals $I \subseteq \mathbb{R}$ such that

$$
\sup _{I} \frac{1}{I} \int_{I} w(t) d t\left(\frac{1}{I} \int_{I} w^{-1 /(p-1)}(t) d t\right)^{p-1} \leq A_{p}
$$

We say that $w \in A_{p}(\mathbb{T})$ if $w$ satisfies (1.2), and we let $A_{p}(w)$ denote the least constant such that (1.2) holds. When $p=1,(1.2)$ is of the form $\sup _{I} \frac{1}{I} \int_{I} w(t) d t$ ess $\sup _{t \in I} \frac{1}{w(t)} \leq A_{1}$.

Theorem 1.1. Let $w$ be a nonnegative $2 \pi$-periodic measurable function. If $1<p<$ $\infty$, then $w \in A_{p}(\mathbb{T})$ if and only if for all $f \in \mathfrak{L}_{p}(\mathbb{T}, w)$,

$$
\left(\int_{-\pi}^{\pi}|\tilde{f}(t)|^{p} w(t) d t\right)^{1 / p} \leq K_{p}\left(\int_{-\pi}^{\pi}|f(t)|^{p} w(t) d t\right)^{1 / p}
$$

where $K_{p}$ is independent of $f$. If $1 \leq p<\infty, w \in A_{p}(\mathbb{T})$ if and only if for all $f \in \mathfrak{L}_{p}(\mathbb{T}, w)$,

$$
\sup _{\tau>0} \tau^{p} \int_{-\pi}^{\pi} \mathbf{1}_{\{t \in[-\pi, \pi):|\tilde{f}(t)|>\tau\}}(t) w(t) d t \leq K_{p}^{p} \int_{-\pi}^{\pi}|f(t)|^{p} w(t) d t
$$

where $K_{p}$ is independent of $f$.

Remark 1.1. We note that from the proof of Theorem 1.1 ([13]), when (1.4) holds, $w \in A_{p}(\mathbb{T})$ with $A_{p}(w)$ less than or equal to $K_{p}^{2}(4 \pi)^{2 p}$. Also, by a modification of the proof in [13], it is enough to assume that (1.4) holds for all $f \in \mathfrak{L}_{p}(\mathbb{T}, w) \cap \mathfrak{L}_{1}(\mathbb{T})$.

Hewitt and Ritter in [8] and [9] make an extensive study of conjugate Fourier series on a-adic solenoids. In this paper, we study weighted norm inequalities on a-adic solenoids $\Sigma_{\mathbf{a}}$. Our main theorem (Theorem 4.4) gives a generalization of Theorem 1.1 in terms of the conjugate function on $\Sigma_{\mathbf{a}}$, obtaining a similar characterization as Hunt et al. [13] of those finite nonnegative Borel measures $\nu$ for which the operator $f \mapsto \tilde{f}$ is bounded from $\mathfrak{L}_{p}\left(\Sigma_{\mathbf{a}}, \nu\right) \cap \mathfrak{L}_{1}\left(\Sigma_{\mathbf{a}}\right)$ into $\mathfrak{L}_{p}\left(\Sigma_{\mathbf{a}}, \nu\right)$.

The plan of the paper is as follows. In Section 2, we give an explicit representation of $\Sigma_{\mathbf{a}}$ and define some other terms needed in our analysis. In Section 3, we show that if $\nu$ is a nonnegative Borel measure on $\Sigma_{\mathbf{a}}$, and the operator $f \mapsto \tilde{f}$ is bounded from $\mathfrak{L}_{p}\left(\Sigma_{\mathbf{a}}, \nu\right) \cap \mathfrak{L}_{1}\left(\Sigma_{\mathbf{a}}\right)$ into $\mathfrak{L}_{p}\left(\Sigma_{\mathbf{a}}, \nu\right)$, then $\nu$ is absolutely continuous with respect to Haar measure $\mu$. This shows that we need only characterize those weights $w \in \mathfrak{L}_{1}\left(\Sigma_{\mathbf{a}}\right)$ that satisfy the property that the operator $f \mapsto \tilde{f}$ is bounded from $\mathfrak{L}_{p}\left(\Sigma_{\mathbf{a}}, w\right) \cap \mathfrak{L}_{1}\left(\Sigma_{\mathbf{a}}\right)$ into $\mathfrak{L}_{p}\left(\Sigma_{\mathbf{a}}, w\right)$. In Section 4 , we state and prove a characterization of those weights that satisfy this last property (Theorem 4.4).

\section{Preliminaries}

2.1. The a-adic solenoid and its character group. Up to isomorphism, any non-cyclic subgroup of $\mathbb{Q}$ can be described as follows. Let $\mathbf{a}=\left(a_{0}, a_{1}, \ldots\right)$ be a fixed infinite sequence of integers all greater than 1 . Let

$$
A_{0}=1, A_{1}=a_{0}, A_{2}=a_{0} a_{1}, \ldots, A_{n}=a_{0} a_{1} \cdots a_{n-1}, \ldots
$$

Let $\mathbb{Q}_{\mathbf{a}}$ be the set of all rational numbers $\frac{l}{A_{k}}$, where $l \in \mathbb{Z}$ and $k \in \mathbb{Z}^{+}$. Clearly $\mathbb{Q}_{\mathbf{a}}$ is a non-cyclic additive subgroup of $\mathbb{Q}$, and as shown in [2], any non-cyclic subgroup of $\mathbb{Q}$ is of this form.

According to the Pontrjagin duality ([10, 24.8, p. 378]), the character group of $\mathbb{Q}_{\mathbf{a}}$ is a compact abelian group, which we denote by $\Sigma_{\mathbf{a}}$, and the character group of $\Sigma_{\mathbf{a}}$ is again $\mathbb{Q}_{\mathbf{a}}$. We let $\mu$ denote normalized Haar measure on $\Sigma_{\mathbf{a}}$. The group $\Sigma_{\mathbf{a}}$ can be realized as the set $[0,1) \times \Delta_{\mathbf{a}}$, which is described in detail in $[10$, Section 
10]. The group $\Delta_{\mathbf{a}}$ consists of all infinite sequences $\mathbf{x}=\left(x_{0}, x_{1}, \ldots, x_{k}, \ldots\right)$ where each $x_{k} \in\left\{0,1, \ldots, a_{k}-1\right\}$. Addition in $\Delta_{\mathbf{a}}$ is defined coordinate-wise and carrying quotients (see $[10,10.2]$ ). Also, the elements $\mathbf{u}=(1,0,0, \ldots)$ and $\mathbf{0}=(0,0,0, \ldots)$ are both in $\Delta_{\mathbf{a}}$, and addition on $[0,1) \times \Delta_{\mathbf{a}}$ is defined by

$$
(\xi, \mathbf{x})+(\eta, \mathbf{y})=(\xi+\eta-\lfloor\xi+\eta\rfloor, \mathbf{x}+\mathbf{y}-\lfloor\xi+\eta\rfloor \mathbf{u})
$$

where $\lfloor\cdot\rfloor$ is the greatest integer function. The group $\Sigma_{\mathbf{a}}$ is a compact connected Abelian group admitting a continuous homomorphism $\varphi: \mathbb{R} \rightarrow \Sigma_{\mathbf{a}}$, where $\varphi(\mathbb{R})$ is a dense subgroup of $\Sigma_{\mathbf{a}}$ and

$$
\varphi(s)=(s-\lfloor s\rfloor,\lfloor s\rfloor \mathbf{u})
$$

([10, Theorem 10.13], and $[9,3.2])$. For $k=1,2, \ldots$, define the sets

$$
\Lambda_{k}=\left\{(0, \mathbf{x}) \in \Sigma_{\mathbf{a}}: x_{0}=x_{1}=\cdots=x_{k-1}=0\right\} .
$$

The sets $\Lambda_{k}$ are compact, closed subgroups of $\Sigma_{\mathbf{a}}$ ([10, Theorem 10.5, p. 110]), and we let $\mu_{k}$ denote normalized Haar measure on $\Lambda_{k}$. The measure $\mu_{k}$ is a singular Borel measure on $\Sigma_{\mathbf{a}}$, and the Fourier transform is equal to the indicator function of $\left(1 / A_{k}\right) \mathbb{Z}:$

$$
\widehat{\mu}_{k}=\mathbf{1}_{\left(1 / A_{k}\right) \mathbb{Z}}
$$

$([9,5.1 \mathrm{ff}$, p. 825$])$. For all $k \in \mathbb{N}$, the quotient group $\Sigma_{\mathbf{a}} / \Lambda_{k}$ is topologically isomorphic to the circle group $\mathbb{T}$ (see $[8,3.1]$ ). Indeed, the mapping

$$
\pi_{k}(t, \mathbf{x})=\chi_{\frac{1}{A_{k}}}(t, \mathbf{x})
$$

is a continuous homomorphism of $\Sigma_{\mathbf{a}}$ onto $\mathbb{T}$ with kernel $\Lambda_{k}$ where

$$
\chi_{\frac{1}{A_{k}}}((t, \mathbf{x}))=\exp \left(2 \pi i \frac{1}{A_{k}}\left(t+\sum_{h=0}^{k-1} x_{h} A_{h}\right)\right)
$$

is the character corresponding to the element $\frac{1}{A_{k}}$ of $\mathbb{Q}_{\mathbf{a}}$. Also, if $f \in \mathfrak{L}_{1}\left(\Sigma_{\mathbf{a}}\right)$ and $f$ is constant on cosets of $\Lambda_{k}$, then $f=f * \mu_{k}$, and there is a function $f_{k} \in \mathfrak{L}_{1}(\mathbb{T})$ that satisfies $f=f * \mu_{k}=f_{k} \circ \pi_{k}$ and

$$
\int_{\Sigma_{\mathbf{a}}} f d \mu=\int_{\Sigma_{\mathbf{a}}} f_{k} \circ \pi_{k} d \mu=\int_{\mathbb{T}} f_{k} d x
$$

$([11,28.55]$ and $[9,5.1 .3])$

Martingales on $\Sigma_{\mathbf{a}}$. If $f \in \mathfrak{L}_{1}\left(\Sigma_{\mathbf{a}}\right)$, then the sequence $\left(f * \mu_{k}\right)_{k \geq 0}$ is a martingale relative to a sequence of $\sigma$-algebras $\left(\mathfrak{F}_{k}\right)_{k \geq 0}$ where $\mathfrak{F}_{k}$ consists of those Borel sets $F \subset \Sigma_{\mathbf{a}}$ such that $F+\Lambda_{k}=F$ (see [6, Theorem 5.4.1]). The functions $f * \mu_{k}$ also are known as the conditional expectations of $f$ relative to $\mathfrak{F}_{k}$. It is a well-known theorem of Doob's that if $f \in \mathfrak{L}_{p}\left(\Sigma_{\mathbf{a}}\right)$, then $f * \mu_{k} \rightarrow f$ in $\mathfrak{L}_{p}\left(\Sigma_{\mathbf{a}}\right)$ as $k \rightarrow \infty$ (see [5], or [6, Theorem 5.2.6]).

The conjugate function on $\Sigma_{\mathbf{a}}$. It is easy to see that $\mathbb{Q}_{\mathbf{a}}$ admits exactly one order $P$ under which 1 is in $P$; the order is the one inherited from the usual order on $\mathbb{R}$. We take this ordering on $\mathbb{Q}_{\mathbf{a}}$ where $P=\left\{\chi_{\alpha} \in \mathbb{Q}_{\mathbf{a}}: \alpha \geq 0\right\}$. For $f \in \mathfrak{L}_{2}\left(\Sigma_{\mathbf{a}}\right)$, we use the Fourier transform and the order generated by $P$ to define the conjugate function $\tilde{f}$ :

$$
\tilde{f}^{\wedge}\left(\chi_{\alpha}\right)=-i \operatorname{sgn}_{P}\left(\chi_{\alpha}\right) \hat{f}\left(\chi_{\alpha}\right)\left(\chi_{\alpha} \in \mathbb{Q}_{a}\right)
$$

where $\operatorname{sgn}_{P}\left(\chi_{\alpha}\right)=-1,0$, or 1 , according to $\alpha<0, \alpha=0$, or $\alpha>0$, respectively. As noted before, if $1<p<\infty$, the operator $f \mapsto \tilde{f}$ extends from $\mathfrak{L}_{2}\left(\Sigma_{\mathbf{a}}\right) \cap \mathfrak{L}_{p}\left(\Sigma_{\mathbf{a}}\right)$ 
to a bounded linear operator of $\mathfrak{L}_{p}\left(\Sigma_{\mathbf{a}}\right)$ ([1, Theorem 7.2]). In addition, the conjugate function $\tilde{f}$ has an integral representation that exists $\mu$-almost everywhere for all functions $f \in \mathfrak{L}_{1}\left(\Sigma_{\mathbf{a}}\right)([1,6.11(\mathrm{c})$ and Theorem 6.5]).

The ergodic $A_{p}$-condition on $\Sigma_{\mathbf{a}}$. Let $\varphi: \mathbb{R} \rightarrow \Sigma_{\mathbf{a}}$ be the continuous homomorphism defined in (2.1). If $1 \leq p<\infty$ and $w$ is a nonnegative function in $\mathfrak{L}_{1}\left(\Sigma_{\mathbf{a}}\right)$, we say that $w$ is in $A_{p}\left(\Sigma_{\mathbf{a}}\right)$ if the following condition is satisfied: for almost every $x \in \Sigma_{\mathbf{a}}$,

$$
\sup _{I} \frac{1}{|I|} \int_{I} w(x-\varphi(t)) d t\left(\frac{1}{|I|} \int_{I} w^{-1 /(p-1)}(x-\varphi(t)) d t\right)^{p-1} \leq K_{p}
$$

where $K_{p}$ is a constant independent of $x$. We let $A_{p}(w)$ denote the least constant such that (2.4) holds.

\section{The continuity of the conjugate function with respect to Borel measures}

In this section, we show that if $\nu$ is a finite nonnegative Borel measure on $\Sigma_{\mathbf{a}}$, the continuity of the operator $f \mapsto \tilde{f}$ from $\mathfrak{L}_{p}\left(\Sigma_{\mathbf{a}}, \nu\right) \cap \mathfrak{L}_{1}\left(\Sigma_{\mathbf{a}}\right)$ into $\mathfrak{L}_{p}\left(\Sigma_{\mathbf{a}}, \nu\right)$ implies that $\nu \ll \mu$ where $\mu$ is Haar measure on $\Sigma_{\mathbf{a}}$.

Theorem 3.1. Let $1<p<\infty$. Let $\nu$ be a finite nonnegative Borel measure on $\Sigma_{\mathbf{a}}$. Suppose that the inequality

$$
\|\tilde{f}\|_{\mathcal{L}_{p}\left(\Sigma_{\mathbf{a}}, \nu\right)} \leq K_{p}\|f\|_{\mathfrak{L}_{p}\left(\Sigma_{\mathbf{a}}, \nu\right)}
$$

is valid for all $f \in \mathfrak{L}_{p}\left(\Sigma_{\mathbf{a}}, \nu\right) \cap \mathfrak{L}_{1}\left(\Sigma_{\mathbf{a}}\right)$ where $K_{p}$ is independent of $f$. Then $\nu \ll \mu$, and hence there is a nonnegative function $w$ in $\mathfrak{L}_{1}\left(\Sigma_{\mathbf{a}}\right)$ such that $d \nu=w d \mu$.

Proof. Assuming that the linear operator $f \mapsto \tilde{f}$ is bounded from $\mathfrak{L}_{p}\left(\Sigma_{\mathbf{a}}, \nu\right) \cap \mathfrak{L}_{1}\left(\Sigma_{\mathbf{a}}\right)$ into $\mathfrak{L}_{p}\left(\Sigma_{\mathbf{a}}, \nu\right)$, we can continuously extend the operator to all of $\mathfrak{L}_{p}\left(\Sigma_{\mathbf{a}}, \nu\right)$. Let $T$ denote the extended linear operator. Fix a real-valued function $g$ in $\mathfrak{L}_{q}\left(\Sigma_{\mathbf{a}}, \nu\right) \cap \mathfrak{L}_{1}\left(\Sigma_{\mathbf{a}}\right)$ where $\frac{1}{p}+\frac{1}{q}=1$. Then by Hölder's inequality, we have for all $f \in \mathfrak{L}_{p}\left(\Sigma_{\mathbf{a}}, \nu\right)$,

$$
\left|\int_{\Sigma_{\mathbf{a}}}(T f) g d \nu\right| \leq\|T f\|_{\mathfrak{L}_{p}\left(\Sigma_{\mathbf{a}}, \nu\right)}\|g\|_{\mathfrak{L}_{q}\left(\Sigma_{\mathbf{a}}, \nu\right)} \leq K_{p}\|g\|_{\mathcal{L}_{q}\left(\Sigma_{\mathbf{a}}, \nu\right)}\|f\|_{\mathfrak{L}_{p}\left(\Sigma_{\mathbf{a}}, \nu\right)} .
$$

Hence, if we define the linear functional $L_{g}: \mathfrak{L}_{p}\left(\Sigma_{\mathbf{a}}, \nu\right) \rightarrow \mathbb{C}$ by $L_{g} f=\int_{\Sigma_{\mathbf{a}}}(T f) g d \nu$, then $L_{g}$ is bounded. By the Riesz Representation Theorem ([15, p. 284]), there is a function $h \in \mathfrak{L}_{q}\left(\Sigma_{\mathbf{a}}, \nu\right)$ such that

$$
L_{g} f=\int_{\Sigma_{\mathbf{a}}}(T f) g d \nu=\int_{\Sigma_{\mathbf{a}}} h f d \nu
$$

for all $f \in \mathfrak{L}_{p}\left(\Sigma_{\mathbf{a}}, \nu\right)$.

We claim that $h$ is real-valued $\nu$-a.e. To see this, consider a continuous character $\chi_{\alpha} \in P \backslash\{0\}$ (so then $\alpha>0$ and $\bar{\chi}_{\alpha} \in(-P) \backslash\{0\}$ ). By (3.2), we have

$$
\begin{aligned}
\int_{\Sigma_{\mathbf{a}}}\left(\operatorname{Re} \chi_{\alpha}\right) h d \nu & =\frac{1}{2} \int_{\Sigma_{\mathbf{a}}}\left(\chi_{\alpha}+\bar{\chi}_{\alpha}\right) h d \nu=\frac{1}{2} \int_{\Sigma_{\mathbf{a}}}\left(T \chi_{\alpha}+T \bar{\chi}_{\alpha}\right) g d \nu \\
& =\frac{1}{2} \int_{\Sigma_{\mathbf{a}}}\left(-i \chi_{\alpha}+i \bar{\chi}_{\alpha}\right) g d \nu \\
& =\int_{\Sigma_{\mathbf{a}}}\left(\operatorname{Im} \chi_{\alpha}\right) g d \nu
\end{aligned}
$$

Since the last integral is real-valued, $\int_{\Sigma_{\mathbf{a}}} \operatorname{Re} \chi_{\alpha} \operatorname{Im} h d \nu=0$. Similarly, $\int_{\Sigma_{\mathbf{a}}} \operatorname{Im} \chi_{\alpha}$ $\operatorname{Im} h d \nu=0$, so that $\int_{\Sigma_{\mathbf{a}}} \chi_{\alpha} \operatorname{Im} h d \nu=0$. This is also true if $\chi_{\alpha}$ is replaced by any 
trigonometric polynomial $\Sigma_{j=1}^{m} \chi_{\alpha_{j}}\left(\chi_{\alpha_{j}} \in \mathbb{Q}_{a}\right)$. By the Stone-Weierstrass Theorem, the set of trigonometric polynomials on $\Sigma_{\mathbf{a}}$ is dense in the set of all continuous functions on $\Sigma_{\mathbf{a}}$; hence for all continuous functions $f$ on $\Sigma_{\mathbf{a}}$, we have $\int_{\Sigma_{\mathbf{a}}} f \operatorname{Im} h d \nu=0$. But then the signed measure $(\operatorname{Im} h d \nu) \equiv 0$, which means that $\operatorname{Im} h=0 \nu$-a.e. and $h$ is real-valued $\nu$-a.e.

We also claim that $(h+i g) d \nu$ is of analytic type in the sense that $(h+i g) d \nu$ has a Fourier transform vanishing for the negative characters in $\mathbb{Q}_{\mathbf{a}}$ (see $[16$, p. 197]). By (3.2), we have

$$
-i \int_{\Sigma_{\mathbf{a}}} \chi_{\alpha} g d \nu=\int_{\Sigma_{\mathbf{a}}} h \chi_{\alpha} d \nu, \text { for all } \chi_{\alpha} \in P \backslash\{0\}
$$

Thus,

$$
\int_{\Sigma_{\mathbf{a}}} \chi_{\alpha}(h+i g) d \nu=0, \text { for all } \chi_{\alpha} \in P \backslash\{0\}
$$

and equivalently

$$
\int_{\Sigma_{\mathbf{a}}} \bar{\chi}_{\alpha}(h+i g) d \nu=0, \text { for all } \chi_{\alpha} \in(-P) \backslash\{0\} .
$$

Thus, $(h+i g) d \nu$ is of analytic type.

By [12, Theorem 19.42, p. 326], we can write the Lebesgue decomposition of $d \nu$ as $d \nu=d \nu_{s}+d \nu_{a}$ where $d \nu_{s}$ is singular with respect to $d \mu\left(d \nu_{s} \perp d \mu\right)$, and $d \nu_{a}$ is absolutely continuous with respect to $d \mu\left(d \nu_{a} \ll d \mu\right)$. Then it is clear that the Lebesgue decomposition of $(h+i g) d \nu$ is

$$
(h+i g) d \nu=(h+i g) d \nu_{s}+(h+i g) d \nu_{a} .
$$

Since $(h+i g) d \nu$ is of analytic type,

$$
\int_{\Sigma_{\mathbf{a}}}(h+i g) d \nu_{s}=0
$$

([16, Theorem 8.2.3, p. 200]). Since $g$ and $h$ are real-valued $\nu_{s}$-a.e., $\int_{\Sigma_{\mathbf{a}}} g d \nu_{s}=0$. This is true for every continuous real-valued $g \in \mathfrak{L}_{p}\left(\Sigma_{\mathbf{a}}, w\right) \cap \mathfrak{L}_{1}\left(\Sigma_{\mathbf{a}}\right)$, so it is also true for the real and imaginary parts of every continuous complex-valued function $g$, and hence $\nu_{s} \equiv 0$. But then $\nu \ll \mu$, and by the Radon-Nikodym Theorem, ([12, Theorem 19.23 , p. 315]), there is a nonnegative measurable function $w \in \mathfrak{L}_{1}^{+}\left(\Sigma_{\mathbf{a}}\right)$ such that $\nu(A)=\int_{A} w d \mu$ for all Borel measurable subsets $A$ of $\Sigma_{\mathbf{a}}$.

Remark 3.1. We note that the proof of Theorem 3.1 does not depend on the structure of the a-adic solenoid $\Sigma_{\mathbf{a}}$. In fact, using the same argument, we can show that Theorem 3.1 holds for any compact connected abelian group $G$ where the dual is ordered and the conjugate function $\tilde{f}$ is defined as in (1.1).

\section{The $A_{p}$-condition on a-adic solenoids}

We seek to characterize those finite nonnegative Borel measures $\nu$ for which the operator $f \mapsto \tilde{f}$ is bounded from $\mathfrak{L}_{p}\left(\Sigma_{\mathbf{a}}, \nu\right) \cap \mathfrak{L}_{1}\left(\Sigma_{\mathbf{a}}\right)$ into $\mathfrak{L}_{p}\left(\Sigma_{\mathbf{a}}, \nu\right)$. By Theorem 3.1, it suffices to characterize those weights $w \in \mathfrak{L}_{1}\left(\Sigma_{\mathbf{a}}\right)$ for which the operator $f \mapsto \tilde{f}$ is bounded from $\mathfrak{L}_{p}\left(\Sigma_{\mathbf{a}}, w\right) \cap \mathfrak{L}_{1}\left(\Sigma_{\mathbf{a}}\right)$ into $\mathfrak{L}_{p}\left(\Sigma_{\mathbf{a}}, w\right)$. In Theorem 4.4, we show that this property holds if and only if $w$ satisfies the ergodic $A_{p}$-condition in (2.4). 
We prove some propositions before proving Theorem 4.4. It is essential for our analysis to define the following classes of functions for $1 \leq p<\infty$ and $w \in \mathfrak{L}_{1}\left(\Sigma_{\mathbf{a}}\right)$ :

$$
\begin{aligned}
\mathfrak{L}_{p}\left(\Sigma_{\mathbf{a}}, \mu\right) * \mu_{k} & =\left\{f * \mu_{k}: f \in \mathfrak{L}_{p}\left(\Sigma_{\mathbf{a}}\right)\right\} \\
\mathfrak{L}_{p}\left(\Sigma_{\mathbf{a}}, w * \mu_{k}\right) * \mu_{k} & =\left\{f * \mu_{k}: f \in \mathfrak{L}_{p}\left(\Sigma_{\mathbf{a}}, w * \mu_{k}\right)\right\} .
\end{aligned}
$$

From Hewitt and Ross [11, p. 95, Theorem 28.55], $\mathfrak{L}_{p}\left(\Sigma_{\mathbf{a}}\right) * \mu_{k}$ is isometrically isomorphic to $\mathfrak{L}_{p}\left(\Sigma_{\mathbf{a}} / \Lambda_{k}\right) \approx \mathfrak{L}_{p}(\mathbb{T})$. By a modification of the proof in [11], we also have $\left(\mathfrak{L}_{p}\left(\Sigma_{\mathbf{a}}, w * \mu_{k}\right) \cap \mathfrak{L}_{1}\left(\Sigma_{\mathbf{a}}\right)\right) * \mu_{k}$ isometrically isomorphic to $\mathfrak{L}_{p}\left(\mathbb{T}, w_{k}\right) \cap \mathfrak{L}_{1}(\mathbb{T})$ where $w_{k}$ is the function in $\mathfrak{L}_{1}(\mathbb{T})$ such that $w * \mu_{k}=w_{k} \circ \pi_{k}$ (see (2.3)).

We use the following notation. If $\nu$ is a nonnegative Borel measure on $\Sigma_{\mathbf{a}}$ and $1 \leq p<\infty$, we define the Lorentz $\mathfrak{L}_{p, \infty}$ quasi-norm for a measurable function $f$ as

$$
\|f\|_{\mathfrak{L}_{p, \infty}(\nu)}^{*}=\sup _{\tau>0} \tau\left(\nu\left(\left\{x \in \Sigma_{\mathbf{a}}:|f(x)|>\tau\right\}\right)\right)^{1 / p} .
$$

(See $\left[17\right.$, Ch.5, Sect.3]; note that $\|\cdot\|_{\mathfrak{L}_{p, \infty}(\nu)}^{*}$ actually defines a norm when $1<p<\infty$.)

Proposition 4.1. Let $1 \leq p<\infty$. Let $T$ denote the operator $f \mapsto \tilde{f}$, and let $w$ be $a$ nonnegative function in $\mathfrak{L}_{1}\left(\Sigma_{\mathbf{a}}\right)$. Then the following are equivalent:

(i) The inequality

$$
\|T f\|_{\mathfrak{L}_{p, \infty}\left(\Sigma_{\mathbf{a}}, w\right)}^{*} \leq K_{p}\|f\|_{\mathfrak{L}_{p}\left(\Sigma_{\mathbf{a}}, w\right)}
$$

is valid for every $f \in \mathfrak{L}_{p}\left(\Sigma_{\mathbf{a}}, w\right) \cap \mathfrak{L}_{1}\left(\Sigma_{\mathbf{a}}\right)$ where $K_{p}$ is independent of $f$.

(ii) For each $k=1,2, \ldots$, the inequality

$$
\left\|T\left(f * \mu_{k}\right)\right\|_{\mathcal{L}_{p, \infty}\left(\Sigma_{\mathbf{a}}, w * \mu_{k}\right)}^{*} \leq K_{p}\left\|f * \mu_{k}\right\|_{\mathfrak{L}_{p}\left(\Sigma_{\mathbf{a}}, w * \mu_{k}\right)}
$$

is valid for every $f \in \mathfrak{L}_{p}\left(\Sigma_{\mathbf{a}}, w * \mu_{k}\right) \cap \mathfrak{L}_{1}\left(\Sigma_{\mathbf{a}}\right)$ where $K_{p}$ is independent of $f$ and $k$.

Proof. (i) $\rightarrow$ (ii) Let $f$ be a trigonometric polynomial on $\Sigma_{\mathbf{a}}$ and fix an integer $1 \leq k<$ $\infty$. Since $f$ is bounded, $f * \mu_{k}$ is bounded and $f * \mu_{k} \in \mathfrak{L}_{p}\left(\Sigma_{\mathbf{a}}, w\right) \cap \mathfrak{L}_{1}\left(\Sigma_{\mathbf{a}}\right)$. Then we have by Fubini's Theorem and the translation invariance of $\mu$,

$$
\begin{aligned}
\sup _{\tau>0} \tau^{p} & \int_{\Sigma_{\mathbf{a}}} \mathbf{1}_{\left\{x \in \Sigma_{\mathbf{a}}:\left|T\left(f * \mu_{k}\right)(x)\right|>\tau\right\}}(x) w * \mu_{k}(x) d \mu(x) \\
& =\sup _{\tau>0} \tau^{p} \int_{\Sigma_{\mathbf{a}}} \int_{\Sigma_{\mathbf{a}}} \mathbf{1}_{\left\{x \in \Sigma_{\mathbf{a}}:\left|T\left(f * \mu_{k}\right)(x)\right|>\tau\right\}}(x) w(x-y) d \mu_{k}(y) d \mu(x) \\
& =\sup _{\tau>0} \tau^{p} \int_{\Sigma_{\mathbf{a}}} \int_{\Sigma_{\mathbf{a}}} \mathbf{1}_{\left\{x \in \Sigma_{\mathbf{a}}:\left|T\left(f * \mu_{k}\right)(x)\right|>\tau\right\}}(x+y) w(x) d \mu(x) d \mu_{k}(y) \\
& =\sup _{\tau>0} \tau^{p} \int_{\Sigma_{\mathbf{a}}} \int_{\Sigma_{\mathbf{a}}} \mathbf{1}_{\left\{x \in \Sigma_{\mathbf{a}}:\left|T\left(f * \mu_{k}\right)(x+y)\right|>\tau\right\}}(x) w(x) d \mu(x) d \mu_{k}(y)
\end{aligned}
$$

Letting $\left(f * \mu_{k}\right)_{y}$ denote the function $x \mapsto\left(f * \mu_{k}\right)(x+y)$ and applying the hypothesis to $\left(f * \mu_{k}\right)_{y}$, we have from $(4.1)$,

$$
\begin{aligned}
\sup _{\tau>0} \tau^{p} & \int_{\Sigma_{\mathbf{a}}} \mathbf{1}_{\left\{x \in \Sigma_{\mathbf{a}}:\left|T\left(f * \mu_{k}\right)(x)\right|>\tau\right\}}(x) w * \mu_{k}(x) d \mu(x) \\
& \leq K_{p}^{p} \int_{\Sigma_{\mathbf{a}}} \int_{\Sigma_{\mathbf{a}}}\left|\left(f * \mu_{k}\right)_{y}(x)\right|^{p} w(x) d \mu(x) d \mu_{k}(y) \\
& =K_{p}^{p} \int_{\Sigma_{\mathbf{a}}}\left|f * \mu_{k}(x)\right|^{p} w * \mu_{k}(x) d \mu(x) .
\end{aligned}
$$

It is easy to see that this is enough to show that (ii) holds. 
(ii) $\rightarrow$ (i) Consider a trigonometric polynomial $f$ on $\Sigma_{\mathbf{a}}$. Then it is clear that there is an integer $N \geq 1$ such that $f=f * \mu_{k}$ for all $k \geq N$. Fix $\tau>0$. Since $\| w * \mu_{k}-$ $w \|_{\mathfrak{L}_{1}\left(\Sigma_{\mathbf{a}}\right)} \rightarrow 0$, and $f$ is a bounded function, we have by the hypothesis

$$
\begin{aligned}
& \tau^{p} \int_{\Sigma_{\mathbf{a}}} \mathbf{1}_{\left\{x \in \Sigma_{\mathbf{a}}:|T f(x)|>\tau\right\}}(x) w(x) d \mu(x) \\
& \quad=\lim _{\substack{k \rightarrow \infty \\
k \geq N}} \tau^{p} \int_{\Sigma_{\mathbf{a}}} \mathbf{1}_{\left\{x \in \Sigma_{\mathbf{a}}:\left|T\left(f * \mu_{k}\right)(x)\right|>\tau\right\}}(x) w * \mu_{k}(x) d \mu(x) \\
& \quad \leq K_{p}^{p} \lim _{\substack{k \rightarrow \infty \\
k \geq N}} \int_{\Sigma_{\mathbf{a}}}\left|f * \mu_{k}(x)\right|^{p} w * \mu_{k}(x) d \mu(x) \\
& \quad=K_{p}^{p} \int_{\Sigma_{\mathbf{a}}}|f(x)|^{p} w(x) d \mu(x) .
\end{aligned}
$$

It is easy to see that this is enough to show that (i) holds.

Remark 4.1. We note that by slightly modifying the proof of Proposition (4.1), we can show that similar strong-type estimates hold.

Proposition 4.2. Let $1 \leq p<\infty$. Suppose $w$ is a nonnegative function in $\mathfrak{L}_{1}\left(\Sigma_{\mathbf{a}}\right)$ and $w$ is constant on the cosets of $\Lambda_{k}$ for some positive integer $k$. Let $w_{k}$ denote the function in $\mathfrak{L}_{1}(\mathbb{T})$ such that $w=w * \mu_{k}=w_{k} \circ \pi_{k}$ (see (2.3)). Then $w$ is in $A_{p}\left(\Sigma_{\mathbf{a}}\right)$ if and only if $w_{k}$ is in $A_{p}(\mathbb{T})$. Moreover, in this case $A_{p}\left(w_{k}\right) \leq A_{p}(w)$.

Proof. We show that the necessity part of the proposition holds. The sufficiency part follows by a similar argument. Assume that $w$ is in $A_{p}\left(\Sigma_{\mathbf{a}}\right)$ with bound $A_{p}(w)$. Let $I=(a, b)$ be an interval in $\mathbb{R}$. Let $(t, \mathbf{x})$ be an element in $\Sigma_{\mathbf{a}}$ such that (2.4) holds. As noted in (2.2) and the following, we have $\pi_{k}((t, \mathbf{x}))=\chi_{\frac{1}{A_{k}}}((t, \mathbf{x}))=\exp \left(2 \pi i \frac{1}{A_{k}} t_{0}\right)$ where $t_{0}=t+\sum_{h=0}^{k-1} x_{h} A_{h}$. We consider the expression

$$
\frac{1}{|I|} \int_{I} w_{k}(\exp (i s)) d s\left(\frac{1}{|I|} \int_{I} w_{k}^{-1 /(p-1)}(\exp (i s)) d s\right)^{p-1}
$$

Let $s=\frac{2 \pi}{A_{k}}\left(t_{0}-u\right), d s=-\frac{2 \pi}{A_{k}} d u, a^{\prime}=t_{0}-\frac{A_{k}}{2 \pi} a, b^{\prime}=t_{0}-\frac{A_{k}}{2 \pi} b$, and $I^{\prime}=\left(b^{\prime}, a^{\prime}\right)$. It is easily observed that $\pi_{k}(\varphi(u))=\chi_{\frac{1}{A_{k}}}(\varphi(u))=\exp \left(2 \pi i \frac{1}{A_{k}} u\right.$ ) for all $u \in \mathbb{R}$ (see $[9,3.2 .4$ ff]). Since $w$ is in $A_{p}\left(\Sigma_{\mathbf{a}}\right)$ with bound $A_{p}(w)$ and $w=w * \mu_{k}=w_{k} \circ \pi_{k}$, we can use a change of variables to see that (4.2) is bounded by $A_{p}(w)$ :

$$
\begin{aligned}
& \frac{1}{|I|} \int_{I} w_{k}(\exp (i s)) d s\left(\frac{1}{|I|} \int_{I} w_{k}^{-1 /(p-1)}(\exp (i s)) d s\right)^{p-1} \\
& =\frac{1}{|I|}\left(\frac{-2 \pi}{A_{k}}\right) \int_{a^{\prime}}^{b^{\prime}} w_{k}\left(\exp \left(2 \pi i \frac{1}{A_{k}}\left(t_{0}-u\right)\right)\right) d u \\
& \quad \times\left(\frac{1}{|I|} \frac{-2 \pi}{A_{k}} \int_{a^{\prime}}^{b^{\prime}} w_{k}^{-1 /(p-1)}\left(\exp \left(2 \pi i \frac{1}{A_{k}}\left(t_{0}-u\right)\right)\right) d u\right)^{p-1} \\
& =\frac{1}{\left|I^{\prime}\right|} \int_{I^{\prime}} w_{k}\left(\pi_{k}((t, \mathbf{x})-\varphi(u))\right) d u \\
& \quad \times\left(\frac{1}{\left|I^{\prime}\right|} \int_{I^{\prime}} w_{k}^{-1 /(p-1)}\left(\pi_{k}((t, \mathbf{x})-\varphi(u))\right) d u\right)^{p-1} \\
& \leq A_{p}(w) .
\end{aligned}
$$


This is true for any interval $I$, hence $w_{k}$ is in $A_{p}(\mathbb{T})$ with bound less than or equal to $A_{p}(w)$.

The next proposition shows that if $w \in A_{p}\left(\Sigma_{\mathbf{a}}\right)$, then the operator $f \mapsto \tilde{f}$ is bounded from $\mathfrak{L}_{p}\left(\Sigma_{\mathbf{a}}, w\right) \cap \mathfrak{L}_{1}\left(\Sigma_{\mathbf{a}}\right)$ into $\mathfrak{L}_{p}\left(\Sigma_{\mathbf{a}}, w\right)$. The proof is similar to that of [14, Theorem 2.1 and Corollary 2.4], using the transference methods of Coifman and Weiss [4]. We include the proof for completeness.

Proposition 4.3. Let $T$ denote the operator $f \mapsto \tilde{f}$ and let $w$ be a nonnegative function in $\mathfrak{L}_{1}\left(\Sigma_{\mathbf{a}}\right)$. If $1<p<\infty$ and $w \in A_{p}\left(\Sigma_{\mathbf{a}}\right)$, then the inequality

$$
\|T f\|_{\mathcal{L}_{p}\left(\Sigma_{\mathbf{a}}, w\right)} \leq A_{p}(w)\|f\|_{\mathcal{L}_{p}\left(\Sigma_{\mathbf{a}}, w\right)}
$$

is valid for all $f \in \mathfrak{L}_{p}\left(\Sigma_{\mathbf{a}}, w\right) \cap \mathfrak{L}_{1}\left(\Sigma_{\mathbf{a}}\right)$, where $A_{p}(w)$ is independent of $f$. If $p=1$ and $w \in A_{1}\left(\Sigma_{\mathbf{a}}\right)$, then the inequality

$$
\|T f\|_{\mathfrak{L}_{1, \infty}\left(\Sigma_{\mathbf{a}}, w\right)}^{*} \leq A_{1}(w)\|f\|_{\mathfrak{L}_{1}\left(\Sigma_{\mathbf{a}}, w\right)}
$$

is valid for all $f \in \mathfrak{L}_{1}\left(\Sigma_{\mathbf{a}}, w\right) \cap \mathfrak{L}_{1}\left(\Sigma_{\mathbf{a}}\right)$, where $A_{1}(w)$ is independent of $f$.

Proof. We show the proposition holds for the case $1<p<\infty$. The case $p=1$ follows by a similar argument. We assume that $w \in A_{p}\left(\Sigma_{\mathbf{a}}\right)$ with bound $A_{p}(w)$ and show that the inequality

$$
\|T f\|_{\mathcal{L}_{p}\left(\Sigma_{\mathbf{a}}, w\right)} \leq A_{p}(w)\|f\|_{\mathfrak{L}_{p}\left(\Sigma_{\mathbf{a}}, w\right)}
$$

is valid for all $f \in \mathfrak{L}_{p}\left(\Sigma_{\mathbf{a}}, w\right) \cap \mathfrak{L}_{1}\left(\Sigma_{\mathbf{a}}\right)$. Let $K_{n}=\left\{t: \frac{1}{n} \leq|t| \leq n\right\}$ and $k_{n}(t)=$ $\frac{1}{\pi t} \mathbf{1}_{K_{n}}(t)$ and $H_{n} f(x)=\int_{\mathbb{R}} f(x-\varphi(t)) k_{n}(t) d t$ where $\varphi: \mathbb{R} \rightarrow \Sigma_{\mathbf{a}}$ is the homomorphism defined in (2.1). To see that (4.3) holds, it is enough to show that for all $n \geq 1$, the inequality

$$
\int_{\Sigma_{\mathbf{a}}}\left|H_{n} f(x)\right|^{p} w(x) d \mu(x) \leq A_{p}^{p}(w)\left(1+\frac{1}{n}\right) \int_{\Sigma_{\mathbf{a}}}|f(x)|^{p} w(x) d \mu(x)
$$

is valid for all $f \in \mathfrak{L}_{p}\left(\Sigma_{\mathbf{a}}, w\right) \cap \mathfrak{L}_{1}\left(\Sigma_{\mathbf{a}}\right)$. (By [1, Theorem 6.5 and 6.11(c)], if $f \in \mathfrak{L}_{1}\left(\Sigma_{\mathbf{a}}\right)$, then $\left|H_{n} f(x)\right| \rightarrow|T f(x)|$ for $\mu$-a.e. $x \in \Sigma_{\mathbf{a}}$. So assuming (4.4) holds, we can use Fatou's lemma to show that (4.3) is valid for all $f \in \mathfrak{L}_{p}\left(\Sigma_{\mathbf{a}}, w\right) \cap \mathfrak{L}_{1}\left(\Sigma_{\mathbf{a}}\right)$.)

To see that (4.4) holds, fix $f \in \mathfrak{L}_{p}\left(\Sigma_{\mathbf{a}}, w\right) \cap \mathfrak{L}_{1}\left(\Sigma_{\mathbf{a}}\right)$ and let $n>1$. Since $\mathbb{R}$ is amenable, we can choose a compact set $K$ such that $\frac{\left|K-K_{n}\right|}{|K|}<1+\frac{1}{n}$ (see $[4,2.1$, p. 8]). By the translation invariance of Haar measure $\mu$ and Fubini's theorem, we have

$$
\begin{aligned}
\int_{\Sigma_{\mathbf{a}}} & \left|H_{n} f(x)\right|^{p} w(x) d \mu(x) \\
& =\frac{1}{|K|} \int_{K} \int_{\Sigma_{\mathbf{a}}}\left|H_{n} f(x-\varphi(t))\right|^{p} w(x-\varphi(t)) d \mu(x) d t \\
& =\frac{1}{|K|} \int_{\Sigma_{\mathbf{a}}} \int_{K}\left|\int_{\mathbb{R}} f(x-\varphi(t-s)) k_{n}(s) d s\right|^{p} w(x-\varphi(t)) d t d \mu(x) \\
& =\frac{1}{|K|} \int_{\Sigma_{\mathbf{a}}} \int_{K}\left|\int_{\mathbb{R}} f(x-\varphi(t-s)) \mathbf{1}_{K-K_{n}}(t-s) k_{n}(s) d s\right|^{p} w(x-\varphi(t)) d t d \mu(x) .
\end{aligned}
$$

Let $g_{x}(t)=f(x-\varphi(t)) \mathbf{1}_{K-K_{n}}(t)$ and $w_{x}(t)=w(x-\varphi(t))$. We have assumed that $w \in A_{p}\left(\Sigma_{\mathbf{a}}\right)$, which means that for $\mu$-a.e. $x \in \Sigma_{\mathbf{a}}, w_{x}(t)$ satisfies (2.4) with bound 
$A_{p}(w)$. Then by the above equalities and [13, Theorem 9, p. 247], we have

$$
\begin{aligned}
\int_{\Sigma_{\mathbf{a}}} & \left|H_{n} f(x)\right|^{p} w(x) d \mu(x) \\
& =\frac{1}{|K|} \int_{\Sigma_{\mathbf{a}}} \int_{K}\left|\int_{\mathbb{R}} g_{x}(t-s) k_{n}(s) d s\right|^{p} w_{x}(t) d t d \mu(x) \\
& \leq \frac{A_{p}^{p}(w)}{|K|} \int_{\Sigma_{\mathbf{a}}} \int_{\mathbb{R}}\left|g_{x}(t)\right|^{p} w_{x}(t) d t d \mu(x) \\
& =\frac{A_{p}^{p}(w)}{|K|} \int_{K-K_{n}} \int_{\Sigma_{\mathbf{a}}}|f(x-\varphi(t))|^{p} w(x-\varphi(t)) d \mu(x) d t \\
& =A_{p}^{p}(w) \frac{\left|K-K_{n}\right|}{|K|} \int_{\Sigma_{\mathbf{a}}}|f(x)|^{p} w(x) d \mu(x) .
\end{aligned}
$$

Since $\frac{\left|K-K_{n}\right|}{|K|}<1+\frac{1}{n}$, we have shown that (4.4) holds, completing the proof of the proposition.

Now we state and prove our main theorem.

Theorem 4.4. Let $T$ denote the operator $f \mapsto \tilde{f}$ and let $w$ be a nonnegative function in $\mathfrak{L}_{1}\left(\Sigma_{\mathbf{a}}\right)$. If $1<p<\infty$, then $w \in A_{p}\left(\Sigma_{\mathbf{a}}\right)$ if and only if the inequality

$$
\|T f\|_{\mathfrak{L}_{p}\left(\Sigma_{\mathbf{a}}, w\right)} \leq K_{p}\|f\|_{\mathfrak{L}_{p}\left(\Sigma_{\mathbf{a}}, w\right)}
$$

is valid for all $f \in \mathfrak{L}_{p}\left(\Sigma_{\mathbf{a}}, w\right) \cap \mathfrak{L}_{1}\left(\Sigma_{\mathbf{a}}\right)$, where $K_{p}$ is a constant independent of $f$. If $1 \leq p<\infty$, then $w \in A_{p}\left(\Sigma_{\mathbf{a}}\right)$ if and only if the inequality

$$
\|T f\|_{\mathcal{L}_{p, \infty}\left(\Sigma_{\mathbf{a}}, w\right)}^{*} \leq K_{p}\|f\|_{\mathcal{L}_{p}\left(\Sigma_{\mathbf{a}}, w\right)}
$$

is valid for all $f \in \mathfrak{L}_{p}\left(\Sigma_{\mathbf{a}}, w\right) \cap \mathfrak{L}_{1}\left(\Sigma_{\mathbf{a}}\right)$, where $K_{p}$ is a constant independent of $f$.

Proof. By Proposition (4.3), the necessity parts of the theorem hold. To prove the sufficiency parts of the theorem, let $1 \leq p<\infty$ and assume that (4.6) holds. As noted before, $\left(\mathfrak{L}_{p}\left(\Sigma_{\mathbf{a}}, w * \mu_{k}\right) \cap \mathfrak{L}_{1}\left(\Sigma_{\mathbf{a}}\right)\right) * \mu_{k}$ is isometrically isomorphic to $\mathfrak{L}_{p}\left(\mathbb{T}, w_{k}\right) \cap \mathfrak{L}_{1}(\mathbb{T})$ where $w_{k}$ is a function in $\mathfrak{L}_{1}(\mathbb{T})$ such that $w * \mu_{k}=w_{k} \circ \pi_{k}$. So by Proposition (4.1), for $k=1,2, \ldots$, the inequality

$$
\|T f\|_{\mathfrak{L}_{p, \infty}\left(\mathbb{T}, w_{k}\right)}^{*} \leq K_{p}\|f\|_{\mathfrak{L}_{p}\left(\mathbb{T}, w_{k}\right)}
$$

is valid for all $f \in \mathfrak{L}_{p}\left(\mathbb{T}, w_{k}\right) \cap \mathfrak{L}_{1}(\mathbb{T})$ where $T f$ is the conjugate function of $f$ defined on the circle. By Theorem 1.1, for $k=1,2, \ldots$, we have $w_{k} \in A_{p}(\mathbb{T})$ with bound less than or equal to $K_{p}^{2}(4 \pi)^{2 p}$. By Proposition (4.2), for $k=1,2, \ldots$, we have $w * \mu_{k} \in A_{p}\left(\Sigma_{\mathbf{a}}\right)$ with bound less than or equal to $K_{p}^{2}(4 \pi)^{2 p}$. Fix an interval $I$. Since $\left\|w * \mu_{k}-w\right\|_{\mathcal{L}_{1}\left(\Sigma_{\mathbf{a}}\right)} \rightarrow 0$ as $k \rightarrow \infty$, by Fatou's lemma and Fubini's Theorem, there is a subsequence $\left(w * \mu_{k_{l}}\right)_{l \geq 0}$ such that for $\mu$-a.e. $x$ in $\Sigma_{\mathbf{a}}$, 


$$
\begin{aligned}
\frac{1}{|I|} \int_{I} w(x-\varphi(s)) d s\left(\frac{1}{|I|} \int_{I} w^{-1 /(p-1)}(x-\varphi(s)) d s\right)^{p-1} \\
\leq \frac{1}{|I|} \liminf _{l} \int_{I} w * \mu_{k_{l}}(x-\varphi(s)) d s \\
\quad \times\left(\frac{1}{|I|} \liminf _{l} \int_{I}\left(w * \mu_{k_{l}}\right)^{-1 /(p-1)}(x-\varphi(s)) d s\right)^{p-1} \\
\leq \frac{1}{|I|} \limsup _{l} \int_{I} w * \mu_{k_{l}}(x-\varphi(s)) d s \\
\quad \times \limsup _{l}\left(\frac{1}{|I|} \int_{I}\left(w * \mu_{k_{l}}\right)^{-1 /(p-1)}(x-\varphi(s)) d s\right)^{p-1} \\
=\frac{1}{|I|} \limsup _{l} \int_{I} w * \mu_{k_{l}}(x-\varphi(s)) d s \\
\quad \times\left(\frac{1}{|I|} \int_{I}\left(w * \mu_{k_{l}}\right)^{-1 /(p-1)}(x-\varphi(s)) d s\right)^{p-1} \\
\leq K_{p}^{2}(4 \pi)^{2 p}
\end{aligned}
$$

So, for each interval $I$, the above inequality holds for $x$ in $\Sigma_{\mathbf{a}}$, except possibly on a set of measure 0 (depending on $I$ ). Thus, the inequality holds for $\mu$-a.e. $x$ in $\Sigma_{\mathbf{a}}$ and for all intervals with rational endpoints (countably many). Approximating an arbitrary interval $I$ by an interval with rational endpoints, a straightforward argument shows that the above inequality still holds for $\mu$-a.e. $x$ in $\Sigma_{\mathbf{a}}$ and all intervals $I$, hence showing that (2.4) holds and $w \in A_{p}\left(\Sigma_{\mathbf{a}}\right)$.

Acknowledgment. The work of the authors was supported by a grant from the National Science Foundation.

\section{References}

1. N. Asmar and E. Hewitt, Marcel Riesz's theorem on conjugate Fourier series and its descendants, in Proceedings of the Analysis Conference, Singapore (1986), (Eds. S. T. L. Choy et al.), Elsevier Science Pub., 1988, pp. 1-56.

2. R. A. Beaumont and H. S. Zuckerman, A characterization of the subgroups of the additive rationals, Pacific J. Math. 1 (1951), 169-177.

3. E. Berkson and T. A. Gillespie, The generalized M. Riesz Theorem and transference, Pacific J. Math. 120 (1985), 279-288.

4. R. R. Coifman and G. Weiss, Transference Methods in Analysis, A. M. S. Providence RI, 1977.

5. J. L. Doob, Stochastic Processes, Wiley Publications, New York, 1953.

6. R. E. Edwards and G. I. Gaudry, Littlewood-Paley and Multiplier Theory, Ergeb. Math. Grenzgeb. No. 90, Springer, Berlin, 1977.

7. F. Forelli, The Marcel Riesz theorem on conjugate functions, Trans. Amer. Math. Soc. 106 (1963), 369-390.

8. E. Hewitt and G. Ritter, Fourier series on certain solenoids, Math. Ann. 257 (1981), 61-83.

9. 느, Conjugate Fourier series on certain solenoids, Trans. Amer. Math Soc. 276 (1983), $817-840$.

10. E. Hewitt and K. Ross, Abstract Harmonic Analysis, Vol. 1, Springer-Verlag, 1963.

11. _ Abstract Harmonic Analysis, Vol. 2, Springer-Verlag, 1970.

12. E. Hewitt and K. Stromberg, Real and Abstract Analysis, Springer-Verlag, 1965.

13. R. Hunt, B. Muckenhoupt, and R. Wheeden, Weighted norm inequalities for the conjugate function and Hilbert transform, Trans. Amer. Math. Soc. 176 (1973), 227-251.

14. F. Lancien. Inégalités à poids pour la transformée de Hilbert ergodique et extension à $H^{1}$ ergodique d'un théorème de Bourgain, Séminaire d'Initiation à l'Analyse, (Exp. no. 10, Publ. Math. Univ. Pierre et Marie Curie, 95, Univ. Paris VI, Paris, 1993).

15. H. L. Royden, Real Analysis, 3rd ed., Macmillan Pub. Co., New York, 1988. 
16. W. Rudin, Fourier Analysis on Groups, John Wiley, New York, 1962.

17. E. Stein and G. Weiss, Introduction to Fourier Analysis on Euclidean Spaces, Princeton Univ. Press, Princeton, 1971.

Department of Mathematics, University of Missouri, Columbia, Missouri 65211

E-mail: mathna@mizzou1.missouri.edu, saleem@csulb.edu 